\title{
Les CLA peuvent-ils prévenir l'athérogenèse ?
}

\author{
Jean-Charles MARTIN ${ }^{1}$ \\ Ghislaine AMSLER ${ }^{2}$ \\ Karine VALEILLE ${ }^{2}$ \\ Jacqueline FÉRÉZOU ${ }^{2}$ \\ Michel PARQUET ${ }^{2}$ \\ Daniel GRIPOIS ${ }^{2}$ \\ Annie QUIGNARD-BOULANGÉ ${ }^{3}$ \\ ${ }^{1}$ UMR Inserm 476/Inra 1260, \\ Faculté de Médecine La Timone, \\ 27, Boulevard Jean Moulin, \\ 13360 Marseille Cedex \\ <jean-charles.martin@medecine.univ-mrs.fr> \\ 2 LEN, bâtiment 447, \\ Université Paris-Sud, \\ 91405 Orsay \\ 3 Inserm U465, \\ Centre biomédical des Cordeliers, \\ 15, rue de l'Ecole de Médecine, \\ 75270 Paris Cedex 06
}

Les CLA (acides linoléiques conjugués, ou ALC) constituent une grande famille d'acides gras insaturés en $\mathrm{C} 18$, dont quelques isomères sont des nutriments naturels. Le plus abondant dans I'alimentation humaine est l'acide ruménique (isomère cis9,trans11), apporté par la viande bovine et les produits laitiers, dont il représente un acide gras minoritaire (formé dans le rumen par transformation bactérienne de l'acide linoléique et dans le foie par l'action de la $\Delta 9$ desaturase sur l'acide vaccénique). Par ailleurs, des mélanges synthétiques $d$ 'isomères (le plus souvent trans10, cis12- et cis9, trans11-, $50: 50$ ) sont disponibles dans le commerce.

L'effet des CLA sur les marqueurs de risque de l'athérosclérose et sur l'apparition des lésions vasculaires fait l'objet d'une recherche de mieux en mieux documentée mais les données restent fragmentaires : peu d'études ont examiné l'effet des CLA à la fois sur la survenue de la pathologie et sur les mécanismes physiologiques et moléculaires qui l'accompagnent. $S^{\prime}$ agissant des marqueurs de risque, il faut bien constater la divergence des résultats provenant des études réalisées in vitro, chez l'animal ou chez l'Homme. Ce manque d'unicité dans les réponses biologiques peut s'expliquer par des différences entre les modèles utilisés, par la durée des complémentations, par les interactions des CLA avec d'autres nutriments, et par

\begin{abstract}
The anti-atherogenic properties of CLA are now illustrated in many animal studies (Rabbit, Mice and Hamster) or cell culture experiments. These properties can be observed while using either the CLA isomeric mixture (cis9, trans 11 - and trans 10, cis12-, 50:50) or selected CLA isomers (cis9, trans 11 or trans10, cis12-). Especially, these fatty acids consistently attenuate the outcome of primary vascular lesions (fatty streaks), or can even induce a regression of pre-established lesions. There is, paradoxically, no clear relationship between this beneficial effect and an improvement of the lipid status in blood (triglycerides, total cholesterol, LDL-cholesterol, HDL-cholesterol) usually considered as a hallmark in the etiology of atherosclerosis. On the other hand, recent data obtained both in vitro and in vivo indicate that part of the beneficial effects of CLA occurs through the modulation of vascular inflammatory and oxidant stress factors (cytokines and cyclooxygenase), as well as from the expression of adhesion molecules (VCAM, ICAM) in the aorta. This regulation involves the interaction of CLA with transcription factors such as PPARs, $L X R$ and NF- $K B$ targeting the gene expression dowstream. In human, clinical trials are no more conclusive than the animal experiments with regards to the contribution of CLA to modulate the lipid status in blood. Nevertheless, some data highlight the pro-atherogenic role of CLA and especially of the trans10,cis12-isomer, as demonstrated by the increase in the various markers of oxidative stress (isoprostanes) and inflammatory status (C-reactive protein). In conclusion, whereas there is compelling evidence that feeding mixed or pure isomers of CLA attenuates atherosclerosis initiation and progression in several experimental models, human studies are less convincing. Nevertheless, clinical trials addressing sufficient risk factors and risk markers together are still lacking to draw a definitive conclusion and to set up obvious recommandations. The use of the sole blood lipids do not seem to be appropriate for such an evaluation.
\end{abstract}

Key words: atherosclerosis, CLA, inflammationlipoproteins

l'emploi de formes différentes de ces acides gras. Ainsi, il convient de distinguer les études associant les deux principales formes isomériques de CLA (mélange cis9, trans11- et trans10,cis12-, $50: 50)$ de celles n'utilisant qu'un seul isomère (cis9,trans11- ou trans10,cis12-) dont les effets biologiques ne sont pas nécessairement identiques [1]. De façon intéressante, la plupart des études réalisées chez l'animal (lapin, hamster et souris) pour examiner l'effet sur les lésions précoces (stries lipidiques) concluent toutes (à l'exception d'une étude chez la souris) à un effet de réduction, voire de régression (lapin), des lésions primaires aortiques, et ceci quelle que soit la forme isomérique administrée (tableau 1). Cet effet bénéfique des CLA est dépendant de la dose (dans l'intervalle 0,05 \%$1 \%$ en poids de régime) mais il n'est pas nécessairement associé à une amélioration du taux des lipides circulants (tableau 1). Les études utilisant des isomères simples $n^{\prime}$ apportent pas plus d'informations, si bien qu'aucun consensus ne se dégage sur la spécificité $d^{\prime}$ action de chacun des isomères sur le statut lipidique : les résultats montrent une amélioration (baisse des TG, augmentation du HDL-C et ou baisse du rapport non-HDL-C/HDL-C) ou une aggravation de ce facteur de risque (augmentation des TG, du rapport non-HDL/LDL-
C), ou bien encore aucun effet (tableau 1 et revues dans [1] et [2]). Des études similaires menées chez l'homme n'apportent pas de réponses plus claires sur les marqueurs de risque lipidique (tableau 2).

En fait, l'action des CLA sur les lésions vasculaires précoces ne serait pas systématiquement liée à un effet bénéfique d'un seul ou des deux isomères sur les lipides circulants. Ainsi des études in vitro, utilisant divers modèles cellulaires, ont tenté de mettre en évidence certains mécanismes à la base des propriétés antiathérogènes des CLA. Ces résultats indiquent qu'ils pourraient accélérer l'épuration des LDL par un accroissement de la quantité de LDL-r (hépatocytes de rat, mélange de CLA [3]) et diminuer le recrutement des monocytes par I'endothélium en réduisant l'expression des molécules d'adhésion (cellules Huvec, mélange $d^{\prime}$ 'isomères [4], ou souris, mélange d'isomères [5]), ainsi que la synthèse de médiateurs inflammatoires (macrophages, NO, cytokines $[6,7])$. Ces effets des CLA pourraient s'exercer via l'inhibition de l'activation de NF- $\kappa$ B, comme démontré in vitro (macrophages, mélanges de CLA, [8]). Enfin, s'agissant du risque de thrombose, une étude ex vivo sur plaquettes humaines rapporte une diminution du potentiel d'agrégation en présence des 
Tableau 1. Effets comparatifs des CLA sur les paramètres lipidiques circulant et l'apparition des lésions précoces vasculaires dans différentes espèces.

\begin{tabular}{|c|c|c|}
\hline Espèces & Régimes & Effets plasmatiques et vasculaires \\
\hline $\begin{array}{l}\text { Lapin New Zealand } \\
{[20], \text { Adultes } \delta \& \&}\end{array}$ & $\begin{array}{l}12 \% \text { coco, } 2 \% \text { maïs } / 0.1 \% \text { chol } / 0.5 \% \text { ALC mix } \times 22 \text { sem. } \\
\text { ALC en supplément }\end{array}$ & $\begin{array}{l}\downarrow \text { LDL-C, } \downarrow \text { TG, } \downarrow \text { LDL-C/HDL-C, } \downarrow \text { chol aorte, } \downarrow \text { dévelop. } \\
\text { lésions }\end{array}$ \\
\hline [21], post-sevrés, ơ & $\begin{array}{l}12 \% \text { coco, } 2 \% \text { maïs, } 0,2 \% \text { chol } / 0,1-1 \% \text { ALC mix } \times 13 \text { sem. } \\
\text { ALC en supplément }\end{array}$ & $\begin{array}{l}\uparrow \mathrm{TG}, \uparrow \text { Chol, } \downarrow \text { HDL-C } \\
\downarrow \text { Diminution des lésions préétablies }\end{array}$ \\
\hline [22], Adultes, oे & $\begin{array}{l}13 \% \text { coco, } 1 \% \text { maiss, } 0,2 \% \text { chol } / 0,05-0,5 \% \text { ALCmix } \times 13 \\
\text { sem. } \\
\text { ALC ajoutés contre sucrose }\end{array}$ & $\begin{array}{l}\downarrow \text { ou个 TG selon dose CLA } \\
\downarrow \text { développement lésions dose dépendant }\end{array}$ \\
\hline \multirow[t]{2}{*}{ [23], Adultes, ò } & $\begin{array}{l}\text { Développement des lésions : } \\
13 \% \text { coco, } 1 \% \text { mais, } 0,2 \% \text { chol, } 0,5 \% \text { iso CLA ou } \\
\text { CLAmix } \times 13 \text { sem. } \\
\text { CLA en supplément }\end{array}$ & $\begin{array}{l}9 \mathrm{c}, 11 \mathrm{t}-, 10 \mathrm{t}, 12 \mathrm{c} \text { - et mix :aucun effet sur lipides sériques } \\
\downarrow \text { identique du développement des lésions }\end{array}$ \\
\hline & $\begin{array}{l}\text { Régression lésions pré-établies } \\
6 \% \text { lipide (mais), } 0 \% \text { chol, } 1 \% \text { iso CLA ou CLAmix } \times 13 \\
\text { sem. } \\
\text { CLA ajoutés contre lipides }\end{array}$ & $\begin{array}{l}\text { 10t,12c- et mix : } \downarrow \text { HDL-C, } \uparrow \text { TG } \\
9 c, 11 \mathrm{t}-: \text { aucun effet sur lipides sériques } \\
\downarrow \text { identiques lésions pré-établies }\end{array}$ \\
\hline $\begin{array}{l}\text { Souris (C57BL/6) } \\
{[24], \text { Adultes, } \delta}\end{array}$ & $\begin{array}{l}15 \% \text { lipide (Maïs, olive, MG lait), } 1 \% \text { chol } / 0,5 \% \text { ALC } \\
\text { mix } \times 15 \text { sem. } \\
\text { ALC ajoutés contre } 18: 2\end{array}$ & $\begin{array}{l}\downarrow \text { chol plasm/HDL-C., } \downarrow \text { TG } \\
\uparrow \text { dévelop. Lésions }\end{array}$ \\
\hline $\begin{array}{l}\text { [25], Adultes, oे } \\
\text { apoE-/- }\end{array}$ & $\begin{array}{l}1 \% \text { chol, autres composants non spécifiés } / 1 \% 9 c, 11 \mathrm{t}-\times 8 \\
\text { sem. } \\
9 c, 11 \text { t- en supplément }\end{array}$ & $\begin{array}{l}\downarrow \text { développement des lésions } \\
\downarrow \text { lésions pré-établies } \\
\text { effets systémiques non décrits }\end{array}$ \\
\hline $\begin{array}{l}\text { Hamster syrien doré } \\
\text { [26], adultes ơ } F 1 B\end{array}$ & $\begin{array}{l}11 \% \text { coco+carthame, } 0.12 \% \text { chol/0,06-1,1\% ALC mix x } 11 \\
\text { sem. } \\
\text { ALC en supplément }\end{array}$ & $\begin{array}{l}\downarrow \text { LDL-C+VLDL-C, } \downarrow \text { chol. Plasm., } \downarrow \text { TG } \\
\downarrow \text { dévelop. Lésions }\end{array}$ \\
\hline [27], adultes ơ $F 1 B$ & $\begin{array}{l}13,1 \% \text { palme \& oléisol, } 0,01 \% \text { chol, } 0,6 \% \text { ALCs } \times 8 \text { sem. } \\
9 c, 11 t-, 10 t, 12 c-\text { ou ALC mix ajoutés contre } 18: 2\end{array}$ & $\begin{array}{l}\text { ALC mix \& 10t, } 12 \mathrm{c}-: \downarrow \text { LDL-C, } \downarrow \text { HDL-C, } \downarrow \text { chol, } \uparrow \text { TG } \\
9 c, 11 \mathrm{t}-: \text { Pas d'effet systémique }\end{array}$ \\
\hline [28], post-sevrés ơ Charles River & $\begin{array}{l}10 \% \text { coco hydrogéné, } 0,05 \% \text { chol, } 1 \% \text { ALC mix ou } 0,2 \% \\
9 c, 11 \mathrm{t} \text { - ou } 0,2 \% \text { linoléique } \times 6 \text { sem. } \\
\text { ALC mix, } 9 c, 11 \text { t } \text { et linoléique en supplément }\end{array}$ & $\begin{array}{l}\text { ALC mix : } \downarrow \text { LDL-C+VLDL-C, } \downarrow \text { chol., } \downarrow \text { TC. } \\
9 c, 11 \text { t- : Pas d'effet systémique }\end{array}$ \\
\hline [29], Adultes ơ F1B & $\begin{array}{l}20 \% \text { coco, } 2 \% \text { carthame, } 0,12 \% \text { chol, } 1 \% \text { CLAmix ou } 1 \% \\
\text { linoléique } \times 12 \text { sem. } \\
\text { ALC mix et linoléique en supplément }\end{array}$ & $\begin{array}{l}\uparrow \text { TG } \\
\downarrow \text { développement lésions }\end{array}$ \\
\hline [10], Adultes ơ LPN & $\begin{array}{l}9,4 \% \text { saindoux, } 4,1 \% \text { colza, } 0.05 \% \text { chol, 1,2\% ALC mix ou } \\
0,6 \% 9 c, 11 \mathrm{t}-\times 8 \text { sem. } \\
\text { ALC mix et ruménique en supplément }\end{array}$ & $\begin{array}{l}\text { ALC mix ET 9c, } 11 \mathrm{t}- \\
\downarrow L D L-C, \uparrow \mathrm{HDL}-\mathrm{C}\end{array}$ \\
\hline [30], Adultes ơ Harlan & $\begin{array}{l}10 \% \text { huile palme, } 0,1 \% \text { chol, } 0,5 \% 9 c, 11 \mathrm{t} \text { - ou } 10 \mathrm{t}, 12 \mathrm{c}-\times 6 \\
\text { sem. } \\
\text { ALC en supplément }\end{array}$ & $\begin{array}{l}10 \mathrm{t}, 12 \mathrm{c}-: \\
\downarrow \mathrm{LDL}-\mathrm{C}\end{array}$ \\
\hline [31], post-sevrés ơ Charles River & $\begin{array}{l}14 \% \text { huile de maïs, } 0 \text { ou } 0,3 \% \text { chol, } 1 \% \text { CLA mix } \times 7 \text { sem. } \\
\text { CLA en supplément }\end{array}$ & $\begin{array}{l}\text { sans chol : aucun effet systémique } \\
0,3 \% \text { chol }: \downarrow \text { chol. }\end{array}$ \\
\hline [11], Adultes ôlanvier & $\begin{array}{l}23 \% \text { lipides (dont } 20 \% \text { beurre), } 0,12 \% \text { chol, } 1 \% 9 c, 11 \mathrm{t}- \\
\times 12 \text { sem. } \\
9 c, 11 \text { t-ALC en supplément }\end{array}$ & $\begin{array}{l}\downarrow \text { non-HDL-C / LDL-C } \\
\downarrow \text { développement lésions }\end{array}$ \\
\hline
\end{tabular}

deux isomères majoritaires (cis9, trans 11 - et trans10,cis12-) [9].

Nous avons pu récemment évaluer, chez des hamsters hyperlipémiques recevant un aliment athérogène, la pertinence de certains de ces mécanismes décrits sur des modèles cellulaires. Ainsi, nous avons montré que l'isomère cis 9 , trans 11 - pouvait augmenter la quantité de récepteurs éboueurs hépatiques responsables de l'épuration des lipoprotéines (LDL-r et SR-BI) [10], réduire le rapport non-HDL/HDLcholestérol [10,11], rétablir l'activité plasmatique de la paraoxonase (détoxication des LDL oxydées), abaisser les teneurs d'apoSAA circulante (marqueur du statut inflammatoire) [11] et normaliser, dans l'aorte, l'expression des protéines d'adhésion (VCAM) et des médiateurs pro-inflammatoires (TNF $\alpha$, IL1b, COX-2). Nous avons également pu mesurer dans la paroi de l'aorte un accroissement de l'expression des facteurs de transcription PPAR $\gamma$, PPAR $\alpha$ et LXRa. Ces observations sont compatibles avec un mode d'action des CLA faisant intervenir les boucles de régulation PPARs-NFKB et PPAR $\gamma$-LXR $\alpha$-ABCA1 (figure 1) pour moduler I'inflammation vasculaire et atténuer ainsi la survenue des lésions. De tels effets des CLA (activation de l'expression de PPAR, et répression de NF- $\kappa$ B et des gènes cibles proinflammatoires) ont été récemment rapportés sur un modèle d'entérocolite provoquée chez la souris, autre pathologie à caractère inflammatoire [12].

En opposition avec la plupart des résultats obtenus chez l'animal et sur des modèles cellulaires, un ensemble d'études cliniques menées par le même groupe d'investigateurs indique que le mélange de CLA (trans10, cis12- et cis9, trans11-), l'isomère trans10,cis12- et dans 
Tableau 2. Effets comparatifs des CLA sur les marqueurs lipidiques du risque athérogène dans diverses études d'intervention chez l'humain.

\begin{tabular}{|c|c|c|}
\hline $\begin{array}{l}\text { Références des auteurs et } \\
\text { type de population }\end{array}$ & Type/dose de CLA et durée & Effet systémique \\
\hline [32], ó et $\uparrow, I M C 30$ & 3,4 g CLAmix × $12 \mathrm{sem}$. & Aucun \\
\hline$[33],+$, IMC 23 & 3,9 g CLAmix × 9 sem & $\downarrow \mathrm{TG}$ \\
\hline$[34], \delta, 9, I M C 23$ & $\begin{array}{l}\text { CLAmix } 0,7 \mathrm{~g} \times 4 \text { sem., } \\
\text { puis } 1,4 \mathrm{~g} \times 4 \mathrm{sem} .\end{array}$ & $\downarrow$ HDL-C \\
\hline [35], ơ, IMC 32 & CLAmix 4,2 g × 4 sem. & Aucun \\
\hline$[36], \delta, q, I M C 25$ & CLAmix $4,2 \mathrm{~g} \times 12 \mathrm{sem}$ & $\uparrow$ Chol, $\uparrow$ LDL-C, $\uparrow$ HDL-C \\
\hline$[37], \delta,+, I M C 24$ & $\begin{array}{l}\text { CLAmix } 2 \mathrm{~g} \times 8 \mathrm{sem} . \\
9 \mathrm{c}, 11 \mathrm{t}-1,4 \mathrm{~g} \times 8 \mathrm{sem}\end{array}$ & $\begin{array}{l}\downarrow \text { TG } \\
\downarrow \text { VLDL-C }\end{array}$ \\
\hline [38], ơ, IMC 32 (syndrome X) & $\begin{array}{l}\text { CLAmix } 3,4 \mathrm{~g} \times 4 \mathrm{sem} \\
10 \mathrm{t}, 12 \mathrm{c}-3,4 \mathrm{~g} \times 4 \mathrm{sem}\end{array}$ & $\begin{array}{l}\text { Aucun } \\
\downarrow \text { HDL-C, } \uparrow \text { CRP \& IR }\end{array}$ \\
\hline [17], ơ, IMC 29, diabetiques II & CLAmix $3 \mathrm{~g} \times 8 \mathrm{sem}$ & $\uparrow \mathrm{HDL}-\mathrm{C}, 0 \mathrm{CRP} \& \downarrow$ IS \\
\hline [39], ठ̊, ๆ, IMC 25-30 & $\begin{array}{l}\text { CLAmix-AGL } 3,6 \mathrm{~g} \times 1 \text { an } \\
\text { CLAmix-TC 3,6 } \mathrm{g} \times 1 \text { an }\end{array}$ & $\begin{array}{l}\uparrow \text { Lp(a) et LDL-C } \\
\downarrow \text { HDL-C }\end{array}$ \\
\hline$[40], \delta, I M C 24,5$ & $\begin{array}{l}9 \mathrm{c} 11 \mathrm{t}-0,6-2,4 \mathrm{~g} \times 8 \mathrm{sem} . \\
10 \mathrm{t}, 12 \mathrm{c}-0,6-2,5 \mathrm{~g} \times 8 \mathrm{sem} .\end{array}$ & $\begin{array}{l}\downarrow \text { nonHDL-C et LDL/HDL-C } \\
\uparrow \mathrm{TG}\end{array}$ \\
\hline
\end{tabular}

IMC, indice de masse corporelle $\left(\mathrm{cm} / \mathrm{m}^{2}\right)$; CLAmix, mélange de CLA ; $9 \mathrm{c}, 11 \mathrm{t}$-, acide ruménique ; 10t, 12c-, isomère CLA-10t,12c ; IS, insulino-sensibilité ; IR, insulino-résistance.

une moindre mesure l'isomère cis9, trans11-, ont des effets néfastes sur le stress oxydant (CLAmix, iso trans10, cis12- ou cis9, trans11-) et le statut inflammatoire (CRP circulante) (CLAmix ou iso trans10,cis12-) [13-15], ce que reflète aussi l'augmentation de l'activité de COX-2 [16]. Comme d'autres informations récentes s'avèrent contradictoires [17], il est nécessaire de mener des investigations plus approfondies utilisant des méthodologies différentes, pour conclure sur l'effet exact des CLA sur les mécanismes conduisant à l'athérosclérose.

\section{Conclusion}

En conclusion, les études animales examinant la survenue des signes précoces d'athérosclérose (dépôts lipidiques dans les vaisseaux), tout comme les modèles cellulaires, indiquent que les CLA possèdent un réel potentiel antiathérogène. Cependant, il reste à déterminer

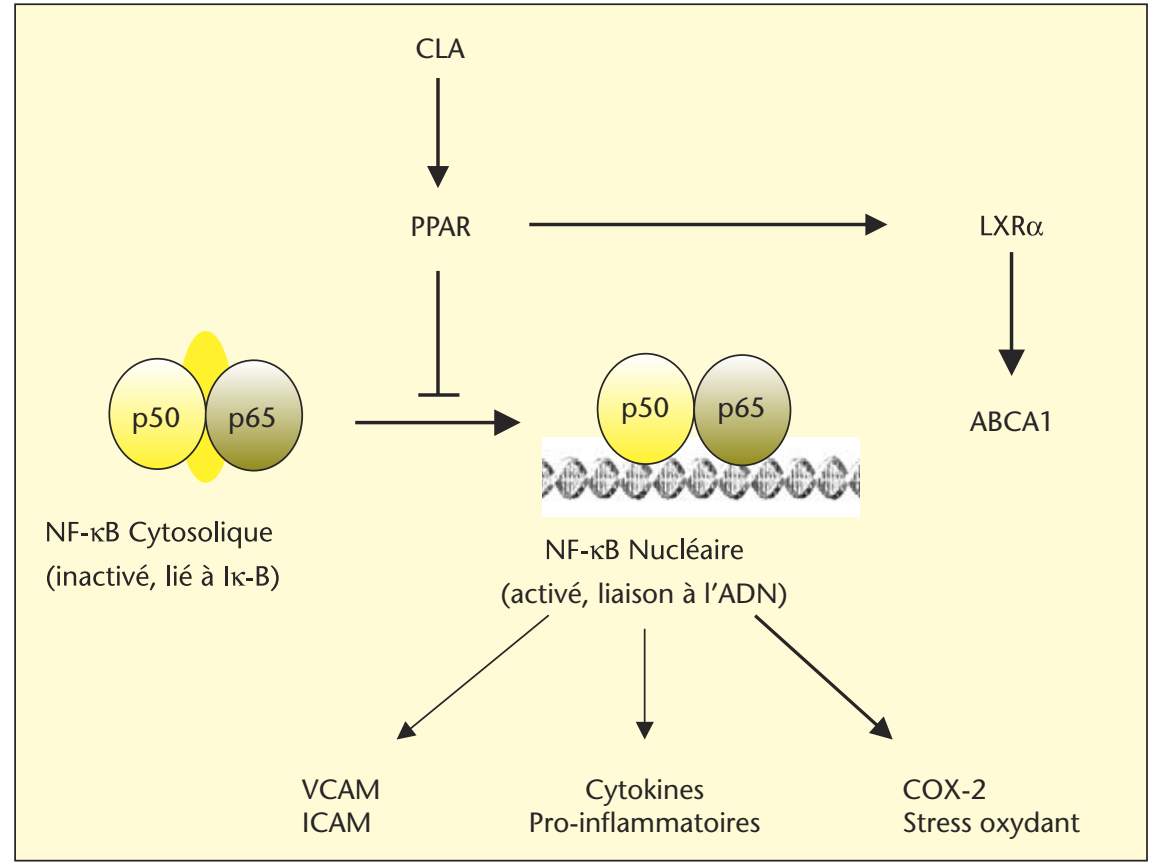

Figure 1. Effet possible des CLA sur les voies d'activation PPAR/NF- $\kappa B$ et PPAR /LXR/ABCA1 (modèle de macrophages murins en culture [68] et aorte de hamster sous régime hypercholestérolémique [11]). plus en détail (au niveau moléculaire) les capacités respectives des différentes formes de CLA disponibles: cis9, trans11-, trans10, cis 12- ou mélange d'isomères), pour établir quelle est la forme la plus avantageuse à administrer. Dans cette perspective, le modèle murin, en raison de sa sensibilité extrême à l'ingestion de CLA (particulièrement à l'isomère trans10,cis12-), doit être utilisé avec circonspection si I'on souhaite l'extrapoler à l'Humain dans le domaine de l'athérosclérose. II apparaît ainsi que le modèle Hamster est beaucoup plus pertinent, car il présente beaucoup d'analogies avec I'Homme sur le plan du métabolisme lipidique. Le modèle porcin présenterait aussi un intérêt certain, mais il n'a fait l'objet jusqu'à présent que de rares études sur les potentialités des CLA dans le domaine de l'athérogenèse [18]. Les essais chez l'Homme visant à évaluer le potentiel anti-athérogène des CLA utilisent fréquemment les lipides circulants comme marqueurs du risque, en raison de leur accessibilité aisée et de leur valeur diagnostique et pronostique reconnue. Toutefois, l'absence d'un consensus concernant l'effet des CLA sur le bilan lipidique, aussi bien chez l'homme que chez l'animal, ne signifie pas que ces acides gras soient d'un intérêt limité dans le cadre de la prévention de l'athérosclérose : il faudrait par exemple tester leur effet chez des individus dont le bilan lipidique est déséquilibré (obèses par exemple), pour pouvoir mettre en évidence un effet des CLA sur la lipémie. L'évaluation du potentiel anti-athérogène des CLA nécessite également de prendre en compte d'autres marqueurs biologiques tels que les marqueurs $d$ 'inflammation et du stress oxydant (CRP, cytokines pro- et anti-inflammatoires, isoprostanes, fractions solubles des protéines d'adhésion, $N F-\kappa B$ ) et I'insulinémie $[13,19]$. Enfin, l'élucidation plus exhaustive des mécanismes d'action au niveau moléculaire reste primordiale pour apprécier le retentissement potentiel (risque ou bénéfice) de la consommation de CLA sur la survenue de la pathologie.

\section{RÉFÉRENCES}

1. MARTIN JC, VALEILLE K. Conjugated linoleic acids: all the same or to everyone its own function? Reprod Nutr Dev $2002 ; 42$ : 525-36.

2. MCLEOD RS, LEBLANC AM, LANGILLE MA, MITCHELL PL, CURRIE DL. Conjugated linoleic acids, atherosclerosis, and hepatic very-lowdensity lipoprotein metabolism. Am / Clin Nutr 2004 ; 79 : 1169S-1174S. 
3. YU-POTHS, YIND, ZHAO G, KRIS ETHERTON PM, ETHERTON TD. Conjugated Linoleic Acid Upregulates LDL Receptor Gene Expression in HepG2 Cells. J Nutr 2004 ; 134 : 68-71.

4. FARQUHARSONA, WU HC, GRANTI, et al. Possible mechanisms for the putative antiatherogenic and antitumorigenic effects of conjugated polyenoic fatty acids. Lipids 1999; 34(Suppl) : S343.

5. CESANO A, VISONNEAU S, SCIMECA JA, KRITCHEVSKY D, SANTOLI D. Opposite effects of linoleic acid and conjugated linoleic acid on human prostatic cancer in SCID mice. Anticancer Res $1998 ; 18$ : 1429-34.

6. YUY, CORRELL PH, VANDEN HEUVEL JP. Conjugated linoleic acid decreases production of pro-inflammatory products in macrophages : evidence for a PPAR[gamma]-dependent mechanism. Biochim Biophys Acta 2002 ; 1581 : 89-99.

7. IWAKIRI Y, SAMPSON DA, ALLEN KG. Suppression of cyclooxygenase-2 and inducible nitric oxide synthase expression by conjugated linoleic acid in murine macrophages. Prostaglandins Leukot Essent Fatty Acids 2002 ; 67 : 435 43.

8. CHENG WL, LII CK, CHEN HW, LIN TH, LIU KL. Contribution of conjugated linoleic acid to the suppression of inflammatory responses through the regulation of the NF-kappaB pathway. J Agric Food Chem 2004 ; 52 : 71-8.

9. TRUITT A, MCNEILL G, VANDERHOEK JY. Antiplatelet effects of conjugated linoleic acid isomers. Biochim Biophys Acta $1999 ; 1438$ : 239 46.

10. VALEILLE K, GRIPOIS D, BLOUQUIT MF, et al. Lipid atherogenic risk markers can be more favourably influenced by the cis-9, trans-11octadecadienoate isomer than a conjugated linoleic acid mixture or fish oil in hamsters. $\mathrm{Br}$ J Nutr $2004 ; 91$ : 191-9.

11. VALEILLE K, FÉRÉZOU J, AMSLER G, et al. A cis 9 trans 11 - conjugated linoeic acid rich-oil reduces the outcome of atherogenic process in hyperlipidemic hamster. Am I Physiol Heart Circ Physiol. In press, 2005

12. BASSAGANYA-RIERAJ, REYNOLDSK,MARTINO CATT S, et al. Activation of PPAR gamma and delta by conjugated linoleic acid mediates protection from experimental inflammatory bowel disease. Gastroenterol $2004 ; 127$ : 777-91.

13. RISERUS U, VESSBY B, ARNLOV I, BASU $S$. Effects of cis-9, trans- 11 conjugated linoleic acid supplementation on insulin sensitivity, lipid peroxidation, and proinflammatory markers in obese men. Am / Clin Nutr 2004 ; 80 : 279-83.
14. BASU S, SMEDMAN A, VESSBY B. Conjugated linoleic acid induces lipid peroxidation in humans. FEBS Lett $2000 ; 468$ : 33-6.

15. BASU S, RISERUS U, TURPEINEN A, VESSBY B. Conjugated linoleic acid induces lipid peroxidation in men with abdominal obesity. Clin Sci (Lond) $2000 ; 99: 511-6$.

16. SMEDMAN A, VESSBY B, BASU S. Isomerspecific effects of conjugated linoleic acid on lipid peroxidation in humans: regulation by alpha-tocopherol and cyclo-oxygenase-2 inhibitor. Clin Sci (Lond) 2004 ; 106 : 67-73.

17. MOLONEY F, YEOW TP, MULLEN A, NOLAN II, ROCHE HM. Conjugated linoleic acid supplementation, insulin sensitivity, and lipoprotein metabolism in patients with type 2 diabetes mellitus. Am / Clin Nutr 2004 ; 80 : 887-95.

18. STANGL GI，MULLER $\mathrm{H}$ ， KIRCHGESSNER M Conjugated linoleic acid effects on circulating hormones, metabolites and lipoproteins, and its proportion in fasting serum and erythrocyte membranes of swine. Eur / Nutr 1999; 38 : 271-7.

19. RISERUS U, BASU S, JOVINGE S, FREDRIKSON GN, ARNLOV J, VESSBY B. Supplementation with conjugated linoleic acid causes isomerdependent oxidative stress and elevated C-reactive protein : a potential link to fatty acid-induced insulin resistance. Circulation $2002 ; 106$ : 1925-9.

20. LEE KN, KRITCHEVSKY D, PARIZA MW. Conjugated linoleic acid and atherosclerosis in rabbits. Atherosclerosis $1994 ; 108$ : 19-25.

21. KRITCHEVSKY D, TEPPER SA, WRIGHT S, TSO P, CZARNECKI SK. Influence of conjugated linoleic acid (CLA) on establishment and progression of atherosclerosis in rabbits. / Am Coll Nutr 2000 ; $19:$ 472S-477S

22. KRITCHEVSKY D, TEPPER SA, WRIGHT S, CZARNECKI SK. Influence of graded levels of conjugated linoleic acid (CLA) on experimental atherosclerosis in rabbits. Nutr Res 2002; 22 : 1275-9.

23. KRITCHEVSKY D, TEPPER SA, WRIGHT S, CZARNECKI SK, WILSON TA, NICOLOSI R]. Conjugated Linoleic Acid Isomer Effects in Atherosclerosis: Growth and Regression of Lesions. Lipids $2004: 39$

24. MUNDAY JS, THOMPSON KG, JAMES KA. Dietary conjugated linoleic acids promote fatty streak formation in the C57BL/6 mouse atherosclerosis model. Br J Nutr $1999 ; 81$ : 251-5.

25. TOOMEY S, ROCHE H, FITZGERALD D, BELTON $\mathrm{O}$. Regression of pre-established atherosclerosis in the apoE-/- mouse by conjugated linoleic acid. Biochem Soc Trans $2003 ; 31$ : 1075-9.
26. NICOLOSI R], ROGERS E], KRITCHEVSKY D, SCIMECA JA, HUTH PJ. Dietary conjugated linoleic acid reduces plasma lipoproteins and early aortic atherosclerosis in hypercholesterolemic hamsters. Artery $1997 ; 22$ : 266-77.

27. DE DECKERE EA, VAN AMELSVOORT JM, MCNEILL GP, JONES P. Effects of conjugated linoleic acid (CLA) isomers on lipid levels and peroxisome proliferation in the hamster. $\mathrm{Br}$ Nutr 1999 ; 82 : 309-17.

28. GAVINO VC, GAVINO G, LEBLANC M], TUCHWEBER $B$. An isomeric mixture of conjugated linoleic acids but not pure cis-9, trans11-octadecadienoic acid affects body weight gain and plasma lipids in hamsters. / Nutr $2000 ; 130: 27-9$.

29. WILSONTA, NICOLOSI RI, CHRYSAM M, KRITCHEVSKY D. Conjugated linoleic acid reduces early aortic atherosclerosis greater than linoleic acid in hypercholesterolemic hamsters. Nutr Res $2000 ; 20: 1795-805$.

30. NAVARRO V, ZABALA A, MACARULLA MT, et al. Effect of conjugated linoleic acid on body fat accumulation and serum lipids in hamsters fed an atherogenic diet. / Physiol Biochem $2003 ; 59: 193-200$

31. SHER I, PRONCZUK A, HAJRI T, HAYES KC. Dietary Conjugated Linoleic Acid Lowers Plasma Cholesterol during Cholesterol Supplementation, but Accentuates the Atherogenic Lipid Profile during the Acute Phase Response in Hamsters. J Nutr $2003 ; 133$ : 456-60.

32. BERVEN G, BYE A, HALS O, et al. Safety of conjugated linoleic acid (CLA) in overweight or obese human volunteers. European Journal of Lipid Science and Technology $2000 ; 102$ : 45562.

33. BENITO P, NELSON G], KELLEY DS, BARTOLINI G, SCHMIDT PC, SIMON V. The effect of conjugated linoleic acid on plasma lipoproteins and tissue fatty acid composition in humans. Lipids $2001 ; 36: 229-36$.

34. MOUGIOS V, MATSAKASA, PETRIDOU A, et al. Effect of supplementation with conjugated linoleic acid on human serum lipids and body fat. / Nutr Biochem $2001 ; 12$ : 585-94.

35. RISERUS U, BERGLUND L, VESSBY B. Conjugated linoleic acid (CLA) reduced abdominal adipose tissue in obese middle-aged men with signs of the metabolic syndrome: a randomised controlled trial. Int / Obes Relat Metab Disord 2001 ; 25 : 1129-35. 
36. SMEDMAN A, VESSBY B. Conjugated linoleic acid supplementation in humans--metabolic effects. Lipids $2001 ; 36: 773-81$.

37. NOONE EJ, ROCHE HM, NUGENT AP, GIBNEY MJ. The effect of dietary supplementation using isomeric blends of conjugated linoleic acid on lipid metabolism in healthy human subjects. $\mathrm{Br}$ J Nutr $2002 ; 88$ : 243-51.
38. RISERUS U, ARNER P, BRISMAR K, VESSBY B. Treatment with dietary trans10cis12 conjugated linoleic acid causes isomer-specific insulin resistance in obese men with the metabolic syndrome. Diabetes Care 2002 ; 25 : 1516-21.

39. GAULLIER JM, HALSE J, HOYE K, et al. Conjugated linoleic acid supplementation for $1 \mathrm{y}$ reduces body fat mass in healthy overweight humans. Am / Clin Nutr 2004 ; 79 : 1118-25.
40. TRICON S, BURDGE GC, KEW S, et al. Opposing effects of cis-9, trans-11 and trans10 ,cis-12 conjugated linoleic acid on blood lipids in healthy humans. Am / Clin Nutr 2004 ; $80: 614-20$. 